\title{
LIMITES DE CONTROLE AJUSTADOS PARA O GRÁFICO DE S COM PARÂMETRO ESTIMADO
}

\author{
Michele Maria da Silva \\ PUC - Rio (DEE). Rua Marquês de São Vicente, 225, Gávea, Rio de Janeiro, RJ. 22451-900. \\ michele.mariadasilva@yahoo.com.br \\ Eugenio Kahn Epprecht \\ PUC - Rio (DEI). Rua Marquês de São Vicente, 225, Gávea, Rio de Janeiro, RJ. 22451-900. \\ eke@puc-rio.br \\ Álvaro de Lima Veiga Filho \\ PUC - Rio (DEE). Rua Marquês de São Vicente, 225, Gávea, Rio de Janeiro, RJ. 22451-900. \\ alvf@ele.puc-rio.br
}

\begin{abstract}
Resumo
Diversas pesquisas mostram que o número de amostras preliminares a serem utilizadas na estimação dos parâmetros para a determinação dos limites de gráficos de controle de processo, de modo que se tenha uma confiança razoável de que a taxa de alarmes falsos não ultrapasse muito o seu valor nominal é proibitivamente alto. Assim, buscando uma alternativa mais viável para a construção do gráfico, são propostos neste artigo limites de controle ajustados para o gráfico de $S$, baseados em dois estimadores usuais para o desviopadrão do processo e tais que a probabilidade de a verdadeira e desconhecida taxa de alarme falso ultrapassar um valor pré-determinado pelo usuário seja pequena e aceitável. Dessa forma, apresentamos um modelo analítico para a determinação dos limites de controle ajustados e, em seguida realizamos uma análise de desempenho do gráfico com limites ajustados na sinalização de aumentos no desvio-padrão do processo, para verificar qual a perda de poder (ou, equivalentemente, qual o aumento no número médio de amostras até o sinal) com o uso destes novos limites.
\end{abstract}

Palavras-chave: Gráfico de controle de $S$; Limites de controle ajustados; Estimação de parâmetros; Fase I; Poder; Alarme Falso.

\begin{abstract}
Several studies show that the number of preliminary samples required for estimating the process parameters and setting control chart limits with a reasonable confidence that the chart's false alarm rate does not largely exceed its nominal value are prohibitively high. For this reason, looking for a more feasible alternative for the construction of the control chart, in this paper adjusted control limits are proposed for the $S$ chart, based on two usual estimators of the standard deviation of the process and such that the probability that the true, unknown, false-alarm rate exceeds a value predetermined by the user is small acceptable. Thus, we present an analytical model to determine the adjusted control limits and next we analyzed the performance of the chart at signalling increases on the process standard deviation, to assess the loss in power (or, equivalently, the increase in the average number of samples until the signal) with the use of these new limits.
\end{abstract}

Keywords: $S$ control chart; Adjusted control limits; Parameter estimation; Phase I; Power; False alarm. 


\section{INTRODUÇÃO}

Os limites dos gráficos de controle são funções dos valores dos parâmetros do processo sob controle, porém caso estes sejam desconhecidos utilizam-se estimativas dos mesmos, obtidas a partir de uma amostra de referência. Pesquisadores têm apontado a deterioração do desempenho dos gráficos de controle como consequência dos efeitos da estimação. Woodall e Montgomery (1999) enfatizaram a importância dos estudos nas seguintes linhas de pesquisa: (1) efeitos da estimação dos parâmetros sobre o desempenho do gráfico; (2) número de amostras que garantam desempenho próximo ao "nominal"; (3) determinação de limites de controle que compensem as perdas com a estimação. A maioria das pesquisas, concentradas no gráfico da média, têm sido desenvolvidas nos dois primeiros pontos e com abordagem na distribuição marginal (incondicional) do número de amostras até o sinal (NAS). A partir de uma abordagem distinta, Chakraborti (2007) - para o gráfico de $\overline{\mathrm{X}}$ e Epprecht et al. (2015) - para o gráfico de S - avaliaram os efeitos da estimação sobre a distribuição condicional do NAS (que é geométrica com parâmetro $p$ desconhecido). Além disso, Epprecht et al. (2015) determinaram o número de amostras $(m)$ tal que a probabilidade de a taxa alarme falso ultrapassar um valor pré-especificado fosse pequena e estabelecida pelo usuário. Segundo essa pesquisa esses números foram bastante elevados.

Motivados por esta questão, neste artigo daremos continuidade à pesquisa realizada por Epprecht et al. (2015). Ao invés do número de amostras, a variável de decisão passa a ser o limite superior de controle do gráfico de $S$. Apresentaremos um modelo analítico através do qual determinamos os limites de controle ajustados avaliados segundo os estimadores $S_{p}$ e $S_{c}$ (definidos na próxima seção) do desvio-padrão e tais que a probabilidade da verdadeira taxa de alarme falso ultrapassar um risco determinado pelo usuário seja pequena. Avaliaremos também o desempenho do gráfico na sinalização de aumentos na dispersão do processo. Assim, o artigo está dividido como segue: na Seção 2 desenvolvemos o modelo analítico; na Seção 3 exibimos e discutimos os resultados dos limites ajustados, analisando o desempenho do gráfico; a síntese dos resultados é apresentada na Seção 4.

\section{O MODELO ANALÍTICO}

A verdadeira e desconhecida probabilidade de alarme falso (ou risco $\alpha_{\text {real }}$ ) bem como a função de distribuição acumulada (fda) para este risco segundo os estimadores $S_{p}$ e $S_{c}$ deduzida por Epprecht et al.(2015) (sob as quais foram analisados os efeitos da estimação) são apresentadas pelas equações 1,2 e 3 , a seguir:

$$
\begin{gathered}
\alpha_{\text {real }}=P\left(\chi_{n-1}^{2}>\frac{k^{2}}{\gamma^{2}} \chi_{n-1, \alpha_{\text {nom }}}^{2} \mid \sigma=\sigma_{0}\right) \\
F_{\alpha_{\text {real }}}(u)=P\left(m(n-1) \frac{S_{p}^{2}}{\sigma^{2}}>m(n-1) \frac{\chi_{n-1, u}^{2}}{\chi_{n-1, \alpha_{\text {nom }}}^{2}}\right)=P\left(\chi_{m(n-1)}^{2}>m(n-1) \frac{\chi_{n-1, u}^{2}}{\chi_{n-1, \alpha_{n o m}}^{2}}\right) \\
F_{\alpha_{\text {real }}}(u)=P\left(\frac{k-\mu_{k}}{\sigma_{k}}>\frac{\sqrt{\chi_{n-1, u}^{2} / \chi_{n-1, \alpha_{\text {nom }}}^{2}}-1}{\sqrt{\left(1-c_{4, n}^{2}\right) / m c_{4, n}^{2}}}\right)=P\left(Z>\frac{\sqrt{\chi_{n-1, u}^{2} / \chi_{n-1, \alpha_{\text {nom }}}^{2}}-1}{\sqrt{\left(1-c_{4, n}^{2}\right) / m c_{4, n}^{2}}}\right)
\end{gathered}
$$

As variáveis $k$ (fator de erro de estimação) e $\gamma$ (fator de aumento do desvio-padrão do processo, que neste caso vale 1), na equação (1) são tais que: 
$k=\frac{\hat{\sigma}_{0}}{\sigma_{0}}\left\{\begin{array}{ll}0<k<1 & \Rightarrow \sigma_{0} \text { foi subestimado } \\ k=1 & \Rightarrow \sigma_{0} \text { estimado sem erro } \\ k>1 & \Rightarrow \sigma_{0} \text { foi superestimado }\end{array} \quad \gamma=\frac{\sigma}{\sigma_{0}}\left\{\begin{array}{l}0<\gamma<1 \\ \gamma=1 \\ \gamma>1\end{array}\right.\right.$

Determinaremos os limites de controle ajustados que garantam a seguinte condição:

$$
P\left(\alpha_{\text {real }}<\alpha_{\text {tol }}\right)=1-p \Leftrightarrow P\left(\alpha_{\text {real }} \geq \alpha_{\text {tol }}\right)=p \text { tal que } \alpha_{\text {tol }}=\left(1+\frac{\varepsilon}{100}\right) \alpha_{\text {nom }}
$$

Vamos inicialmente definir os fatores $L_{n o m}, L_{S_{p}}$ e $L_{S_{c}}$ para após determinar os limites de controle ajustados para o gráfico de $\mathrm{S}$ em função destes fatores. Definamos como fator $L_{\text {nom }}$ o termo $\left(\chi_{n-1, \alpha_{\text {nom }}}^{2} /(n-1)\right)$ presente na equação do LSC para o gráfico de $\mathrm{S}$ (veja Montgomery, 2011):

$$
\begin{array}{|l|l|l}
\hline L S C_{S}=\sqrt{\frac{\chi_{\alpha_{\text {nom }}, n-1}^{2}}{n-1}} & \hat{\sigma}_{0} \quad \text { (5) } & L_{\text {nom }}=\frac{\chi_{\alpha_{\text {nom }}, n-1}^{2}}{n-1} \\
\hline
\end{array}
$$

$\mathrm{O} L S C_{S}$ (que estaremos denotando daqui em diante por $L S C_{\text {nom }}$ ) é função do estimador do desvio-padrão do processo em controle $\left(\sigma_{0}\right)$. Neste trabalho consideraremos dois estimadores do desvio-padrão: o desvio-padrão combinado $S_{p}$ e o estimador não viesado $S_{c}$, calculados por:

\begin{tabular}{|l|l|}
$S_{p}=\sqrt{S_{p}^{2}}=\sqrt{\sum_{i=1}^{m} \frac{(n-1) S_{i}^{2}}{m(n-1)}}=\sqrt{\sum_{i=1}^{m} \frac{S_{i}^{2}}{m}} \quad$ (7) & $S_{c}=\frac{\bar{S}}{c_{4, n}}=\frac{\sum_{i=1}^{m} \frac{S_{i}}{m}}{c_{4, n}}$ \\
\hline
\end{tabular}

onde

$$
S_{i}=\sqrt{S_{i}^{2}}=\sqrt{\frac{1}{n-1} \sum_{j=1}^{n}\left(x_{i j}-\bar{x}_{i}\right)^{2}} ; \quad\left\{\begin{array}{l}
\mu_{S_{i}}=c_{4, n} \sigma \\
\sigma_{S_{i}}=\sqrt{1-c_{4, n}} \sigma
\end{array} \text { com } c_{4, n}=\frac{\Gamma(n / 2)}{\Gamma(n-1 / 2)} \sqrt{\frac{2}{n-1}}\right.
$$

Para a determinação do fator $L_{S_{p}}$ vamos retomar o fda do risco $\alpha_{\text {real }}$ segundo o estimador $S_{p}$ (equação 3) aplicada em $\alpha_{\text {tol }}$ e de forma que a condição dada por (4) seja garantida. Logo:

$$
F_{\alpha_{\text {real }}}\left(\alpha_{\text {tol }}\right)=P\left(\alpha_{\text {real }} \leq \alpha_{\text {tol }}\right)=P\left(\chi_{m(n-1)}^{2}>m(n-1) \frac{\chi_{n-1, \alpha_{\text {tol }}}^{2}}{\chi_{n-1, \alpha_{\text {nom }}}^{2}}\right)=1-p
$$

Pela equação (6) o quantil $\chi_{n-1, \alpha_{n o m}}^{2}$ pode ser escrito como $(n-1) L_{n o m}$, mas como a última equação está em função do estimador $S_{p}$, então este termo será reescrito como $(n-1) L_{S_{p}}$. Logo:

$$
F_{\alpha_{\text {real }}}\left(\alpha_{\text {tol }}\right)=P\left(\chi_{m(n-1)}^{2}>m(n-1) \frac{\chi_{n-1, \alpha_{o l}}^{2}}{(n-1) L_{S_{p}}}\right)=1-p
$$

Nesta última equação, o termo do lado direito da desigualdade representa o $p$-quantil da distribuição qui-quadrado com $m(n-1)$ graus de liberdade, que deixa à sua direita uma área de $(1-p) \%$. Assim o fator $L_{S_{p}}$ pode ser obtido da seguinte maneira:

$$
m(n-1) \frac{\chi_{n-1, \alpha_{t o l}}^{2}}{(n-1) L_{S_{p}}}=\chi_{m(n-1),(1-p)}^{2} \Leftrightarrow L_{S_{p}}=\frac{m(n-1)}{\chi_{m(n-1),(1-p)}^{2}} \frac{\chi_{n-1, \alpha_{t o l}}^{2}}{n-1}
$$


Para a dedução do fator $L_{S_{c}}$, tomamos a fda do risco $\alpha_{\text {real }}$ segundo o estimador $S_{c}$ (equação 8) aplicada em $\alpha_{\text {tol }}$ de forma que a condição dada por (4) seja satisfeita. Assim:

$$
F_{\alpha_{\text {real }}}\left(\alpha_{\text {tol }}\right)=P\left(\alpha_{\text {real }} \leq \alpha_{\text {tol }}\right)=P\left(Z>\frac{\sqrt{\chi_{n-1, \alpha_{\text {tol }}}^{2} / \chi_{n-1, \alpha_{\text {nom }}}^{2}}-1}{\sqrt{\left(1-c_{4, n}^{2}\right) / m c_{4, n}^{2}}}\right)=1-p
$$

Novamente o quantil $\chi_{n-1, \alpha_{\text {nom }}}^{2}$ na equação anterior pode ser escrito como $(n-1) L_{\text {nom }}$, mas como neste caso a fda foi obtida a partir do estimador $S_{c}$, então devemos reescrevê-lo como $(n-1) L_{S_{c}}$. Logo, a última expressão pode ser reescrita como:

$$
1-F_{\alpha_{\text {real }}}\left(\alpha_{\text {tol }}\right)=P\left[Z \leq \frac{\sqrt{\chi_{n-1, \alpha_{\text {tol }} /(n-1) L_{S_{c}}^{2}}^{2}}-1}{\sqrt{\left(1-c_{4, n}^{2}\right) / m c_{4, n}^{2}}}\right]=p
$$

O termo do lado direito da desigualdade representa o p-quantil da distribuição normal padrão, logo ele pode ser reescrito como segue, donde o fator $L_{S_{c}}$ é dado por:

$$
\left(\sqrt{\frac{\chi_{n-1, \alpha_{t o l}}^{2}}{(n-1) L_{S_{c}}}}-1\right) \times\left(\sqrt{\frac{m c_{4, n}^{2}}{\left(1-c_{4, n}^{2}\right)}}\right)=-z_{p} \Leftrightarrow \sqrt{L_{S_{c}}=\frac{1}{\left[1-z_{p} \sqrt{\left(1-c_{4, n}^{2}\right) / m c_{4, n}^{2}}\right]^{2}} \frac{\chi_{n-1, \alpha_{t o l}}^{2}}{n-1}}
$$

Substituindo os fatores $L_{S_{p}}$ e $L_{S_{c}}$ (equações 9 e 10) na equação 5 e denotando-as por $L S C_{S_{p}}$ e $L S C_{S_{c}}$, obtemos os limites de controle ajustados do gráfico de $\mathrm{S}$, como seguem:

$$
\begin{gathered}
L S C_{S_{p}}=\sqrt{\frac{m(n-1)}{\chi_{m(n-1),(1-p)}^{2} \frac{\chi_{n-1, \alpha_{t o l}}^{2}}{n-1}}} \hat{\sigma}_{0} \\
L S C_{S_{c}}=\sqrt{\frac{1}{\left[1-z_{p} \sqrt{\left(1-c_{4, n}^{2}\right) / m c_{4, n}^{2}}\right]^{2}} \frac{\chi_{n-1, \alpha_{t o l}}^{2}}{n-1}} \hat{\sigma}_{0}
\end{gathered}
$$

Como $\forall a>0, P\left(S^{2} \geq a^{2}\right)=P(S \geq a)$, então o poder do gráfico de $\mathrm{S}\left(P d_{S}\right)$ e o número médio de amostras até o alarme verdadeiro (NMA) dos gráficos de $\mathrm{S}^{2}$ e $\mathrm{S}$ são equivalentes. Segundo Montgomery (2011) essas medidas (que denotaremos por nominais) são dadas por:

$$
P d_{S^{2}}=P\left(S^{2}>L S C_{S^{2}}\right)=P\left(S>L S C_{S}\right)=P d_{S} \Rightarrow N M A_{S}=\frac{1}{P d_{S}}
$$

Substituindo os limites ajustados - equações 11 e 12 - na equação13 e após manipulações algébricas, determinamos o $P d_{S_{p}}, P d_{S_{c}}, N M A_{s_{p}}$ e $N M A_{s_{c}}$ cujas equações são:

$$
\begin{gathered}
P d_{S_{p}}=P\left(\chi_{n-1}^{2}>\frac{k^{2}}{\gamma^{2}} \frac{m(n-1)}{\chi_{m(n-1),(1-p)}^{2}} \chi_{n-1, \alpha_{t o l}}^{2}\right) \Rightarrow N M A_{S_{p}}=\frac{1}{P d_{S_{p}}} \\
P d_{S_{c}}=P\left(\chi_{n-1}^{2}>\frac{k^{2}}{\gamma^{2}} \frac{1}{\left[1-z_{p} \sqrt{\left(1-c_{4, n}^{2}\right) / m c_{4, n}^{2}}\right]^{2}} \chi_{n-1, \alpha_{t o l}}^{2}\right) \Rightarrow N M A_{S_{c}}=\frac{1}{P d_{S_{c}}}
\end{gathered}
$$




\section{RESULTADOS E DISCUSSÃO}

Os limites de controle, poderes e NMA's ajustados são avaliados para $m \in\{25$, $50,100,200,500\}, n \in\{5,50,15,20,25,30\}, p \in\{5,10\} \%, \varepsilon \in\{10,20\} \%, \gamma=1,5$ e $\alpha_{\text {nom }}=0,005$.

$\mathrm{Na}$ Tabela 1 as linhas não sombreadas fornecem os valores do $L S C_{S_{p}}$ e do $L S C_{S_{c}}$ e as sombreadas fornecem o "fator de ajuste" do LSC (razão entre o LSC ajustado e o LSC nominal) representado pelo símbolo $\Delta$. Na Tabela 2 as linhas não sombreadas fornecem os poderes ajustados e as sombreadas os NMAs ajustados. Nas Figuras, as linhas tracejadas correspondem aos resultados aplicados ao estimador $S_{c}$ e a linhas contínuas ao estimador $S_{p}$. Vale lembrar que o gráfico é formado por pontos discretos que foram unidos por segmentos de reta para que leitor possa verificar como ocorre a convergência.

De imediato podemos constatar que um aumento nas variáveis $m, p$ e $\varepsilon$ implica na convergência dos limites de controle ajustados, poderes e NMA's para o limite de controle, poder e NMA nominal, respectivamente. Um aumento em $n$ apesar de provocar uma redução tanto nos limites de controle e NMA's ajustados como no limite de controle e NMA nominal ainda garante a convergência (todas limitadas por 1). $\mathrm{O}$ mesmo ocorre com os poderes ajustados e o poder nominal, mas no sentido contrário. Em qualquer caso esse resultado é esperado dado que um aumento em $m$ e $n$ reduz a variabilidade dos estimadores e um aumento em $p$ ou $\varepsilon$ significa maior tolerância a probabilidades de alarme falso distintas da nominal.

A maioria dos manuais de CEP recomenda o uso de 25 a 30 amostras de tamanho entre 4 e 5 para a determinação dos limites de controle, entretanto, qualquer par com $(m \leq 25, n \leq 5)$ resulta em limites de controle, poderes e NMAs ajustados muito distantes dos seus respectivos valores nominais. Para $(p=5 \% ; \varepsilon=10 \%)$ os limites ajustados ultrapassam $12,5 \%$ do limite nominal, implicando em poderes ajustados que correspondem a menos de $49 \%$ do poder nominal ( $N M A s$ ajustados $12,6 \%$ maiores que o $N M A_{\text {hom }}$ ). A situação permanece mesmo duplicando-se os valores de $p$ e $\varepsilon$. Em contrapartida, todo par com $(m \geq 25, n \geq 15)$ retorna limites de controle ajustados que excedem, no máximo, $6 \%$ e $4 \%$ do limite de controle nominal, resultando em poderes ajustados que atingem, no mínimo, 73\% e $81 \%$ do poder nominal, quando adotados o cenário mais conservador e mais otimista para $p$ e $\varepsilon$, respectivamente. A partir $n \geq 15$ obtemos limites de controle, poderes e NMA's ajustados muito próximos aos seus respectivos nominais, seja qual for o $m$ adotado. Nos gráficos podemos notar a proximidade entre as curvas. Neste caso, um aumento no número de observações $(m \times n)$ pode não resultar em grandes ganhos para o usuário. Por exemplo, para ( $m=50 ; n=15)$ os limites de controle ajustados ultrapassam $2,6 \%$ do limite de controle nominal e os poderes ajustados atingem $88 \%$ do poder nominal (NMA's ajustados 14\% maiores que o nominal). Para $(m=100 ; n=15)$ - duplicando-se o número de amostras e, portanto, o número de observações (1.500 contra 750) - obtemos limites de controle ajustados $1,6 \%$ maiores que o limite de controle nominal, resultado em poderes ajustados que perfazem $93 \%$ do poder nominal, se adotarmos $(p=10 \% ; \varepsilon=20 \%)$.

Os valores dos limites de controle ajustados são maiores para os pares $(m, n)$ com $n \geq m$ que os valores dos limites de controle ajustados com $n<m$ sempre que estes pares resultarem no mesmo número de observações. Isto não implica que os limites de controle, poderes e NMA's ajustados no primeiro caso estejam mais próximos ao limite, poder e $N M A$ nominal que no segundo caso, embora esta situação predomine. Os pares $(200,5)$ e $(100,10)$ comprovam isso. Comparando $p$ e $\varepsilon$, podemos notar que um aumento em $p$ torna os limites de controle ajustados mais próximos ao limite de controle nominal que aumento em $\varepsilon$ na mesma proporção, para qualquer combinação com $(m \leq 200$ e $n \leq 30)$. Com outros pares $(m, n)$ o usuário deve fazer uma análise. 
Tabela 1 - Limites de controle ajustados e nominais em função de $m$ e $n$ com $p \in(5 ; 10) \%$; $\varepsilon \in(10 ; 20) \%$ e $\alpha_{\text {nom }}=0,005$.

\begin{tabular}{|c|c|c|c|c|c|c|c|c|c|c|c|c|}
\hline \multicolumn{13}{|c|}{ Limites de controle } \\
\hline \multirow{3}{*}{ ఝ્. } & \multirow{3}{*}{$\mathbf{n}$} & \multirow{3}{*}{$\begin{array}{c}L S C_{n o m} \\
\Delta \\
\forall \mathbf{m}\end{array}$} & \multirow{2}{*}{\multicolumn{5}{|c|}{$L S C_{S_{p}}$}} & \multicolumn{5}{|c|}{$L S C_{S_{c}}$} \\
\hline & & & & & & & & & LSC & / LSC & 1om $(\Delta$ & \\
\hline & & & $\mathrm{m}=\mathbf{2 5}$ & 50 & 100 & 200 & 500 & $m=25$ & 50 & 100 & 200 & 500 \\
\hline \multirow{12}{*}{$\begin{array}{l}\partial \\
\stackrel{\ominus}{\sigma} \\
\| \\
\omega \\
\ddot{n} \\
\| \\
\Xi\end{array}$} & \multirow[t]{3}{*}{5} & 1,927 & 2,167 & 2,086 & 2,032 & 1,996 & 1,965 & 2,173 & 2,090 & 2,035 & 1,998 & 1,966 \\
\hline & & 1 & 1,125 & 1,082 & 1,054 & 1,035 & 1,019 & 1,127 & 1,084 & 1,056 & 1,036 & 1,020 \\
\hline & & 1,619 & 1,746 & 1,704 & 1,675 & 1,655 & 1,638 & 1,747 & 1,705 & 1,676 & 1,656 & 1,639 \\
\hline & 10 & 1 & 1,079 & 1,052 & 1,035 & 1,023 & 1,012 & 1,079 & 1,053 & 1,035 & 1,023 & 1,012 \\
\hline & \multirow[t]{2}{*}{15} & 1,496 & 1,588 & 1,557 & 1,537 & 1,522 & 1,510 & 1,588 & 1,558 & 1,537 & 1,522 & 1,510 \\
\hline & & 1 & 1,062 & 1,041 & 1,027 & 1,018 & 1,009 & 1,062 & 1,041 & 1,027 & 1,018 & 1,009 \\
\hline & \multirow[t]{2}{*}{20} & 1,425 & 1,499 & 1,475 & 1,458 & 1,446 & 1,436 & 1,500 & 1,475 & 1,458 & 1,446 & 1,436 \\
\hline & & 1 & 1,052 & 1,035 & 1,023 & 1,015 & 1,008 & 1,052 & 1,035 & 1,023 & 1,015 & 1,008 \\
\hline & \multirow[t]{2}{*}{25} & 1,378 & 1,441 & 1,420 & 1,406 & 1,396 & 1,387 & 1,441 & 1,420 & 1,406 & 1,396 & 1,387 \\
\hline & & 1 & 1,046 & 1,031 & 1,020 & 1,013 & 1,007 & 1,046 & 1,031 & 1,021 & 1,013 & 1,007 \\
\hline & \multirow[t]{2}{*}{30} & 1,343 & 1,399 & 1,381 & 1,368 & 1,359 & 1,352 & 1,399 & 1,381 & 1,368 & 1,359 & 1,352 \\
\hline & & 1 & 1,042 & 1,028 & 1,018 & 1,012 & 1,006 & 1,042 & 1,028 & 1,019 & 1,012 & 1,006 \\
\hline \multirow{12}{*}{ 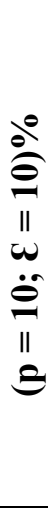 } & \multirow[t]{2}{*}{5} & 1,927 & 2,108 & 2,046 & 2,005 & 1,977 & 1,953 & 2,110 & 2,048 & 2,007 & 1,978 & 1,954 \\
\hline & & 1 & 1,094 & 1,062 & 1,040 & 1,026 & 1,013 & 1,095 & 1,063 & 1,041 & 1,026 & 1,014 \\
\hline & \multirow[t]{2}{*}{10} & 1,619 & 1,715 & 1,683 & 1,660 & 1,645 & 1,632 & 1,715 & 1,683 & 1,661 & 1,646 & 1,632 \\
\hline & & 1 & 1,059 & 1,039 & 1,026 & 1,016 & 1,008 & 1,059 & 1,039 & 1,026 & 1,016 & 1,008 \\
\hline & 15 & 1,496 & 1,565 & 1,542 & 1,526 & 1,515 & 1,505 & 1,565 & 1,542 & 1,526 & 1,515 & 1,505 \\
\hline & & 1 & 1,047 & 1,031 & 1,020 & 1,013 & 1,006 & 1,046 & 1,031 & 1,020 & 1,013 & 1,006 \\
\hline & 20 & 1,425 & 1,481 & 1,462 & 1,449 & 1,440 & 1,432 & 1,481 & 1,462 & 1,449 & 1,440 & 1,432 \\
\hline & & 1 & 1,039 & 1,026 & 1,017 & 1,011 & 1,005 & 1,039 & 1,026 & 1,017 & 1,011 & 1,005 \\
\hline & 25 & 1,378 & 1,426 & 1,410 & 1,398 & 1,391 & 1,384 & 1,426 & 1,410 & 1,399 & 1,391 & 1,384 \\
\hline & & 1 & 1,035 & 1,023 & 1,015 & 1,009 & 1,004 & 1,035 & 1,023 & 1,015 & 1,009 & 1,005 \\
\hline & 30 & 1,343 & 1,386 & 1,371 & 1,362 & 1,355 & 1,349 & 1,385 & 1,371 & 1,362 & 1,355 & 1,349 \\
\hline & & 1 & 1,031 & 1,021 & 1,014 & 1,008 & 1,004 & 1,031 & 1,021 & 1,014 & 1,009 & 1,004 \\
\hline & 5 & 1,927 & 2,153 & 2,072 & 2,018 & 1,982 & 1,951 & 2,158 & 2,076 & 2,021 & 1,984 & 1,953 \\
\hline & & 1 & 1,117 & 1,075 & 1,047 & 1,028 & 1,012 & 1,120 & 1,077 & 1,049 & 1,029 & 1,013 \\
\hline & 10 & 1,619 & 1,737 & 1,695 & 1,666 & 1,647 & 1,630 & 1,738 & 1,696 & 1,667 & 1,647 & 1,630 \\
\hline 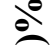 & & 1 & 1,073 & 1,047 & 1,029 & 1,017 & 1,007 & 1,074 & 1,048 & 1,030 & 1,018 & 1,007 \\
\hline ฮి & 15 & 1,496 & 1,581 & 1,551 & 1,530 & 1,516 & 1,503 & 1,581 & 1,551 & 1,530 & 1,516 & 1,503 \\
\hline$\|$ & & 1 & 1,057 & 1,037 & 1,023 & 1,013 & 1,005 & 1,057 & 1,037 & 1,023 & 1,013 & 1,005 \\
\hline y & 20 & 1,425 & 1,494 & 1,469 & 1,452 & 1,441 & 1,431 & 1,494 & 1,469 & 1,453 & 1,441 & 1,431 \\
\hline " & & 1 & 1,048 & 1,031 & 1,019 & 1,011 & 1,004 & 1,048 & 1,031 & 1,019 & 1,011 & 1,004 \\
\hline$\pi$ & 25 & 1,378 & 1,436 & 1,415 & 1,401 & 1,391 & 1,382 & 1,436 & 1,415 & 1,401 & 1,391 & 1,382 \\
\hline & & 1 & 1,042 & 1,027 & 1,017 & 1,010 & 1,003 & 1,042 & 1,027 & 1,017 & 1,010 & 1,003 \\
\hline & 30 & 1,343 & 1,395 & 1,376 & 1,364 & 1,355 & 1,347 & 1,395 & 1,376 & 1,364 & 1,355 & 1,347 \\
\hline & & 1 & 1,038 & 1,025 & 1,015 & 1,009 & 1,003 & 1,038 & 1,025 & 1,015 & 1,009 & 1,003 \\
\hline & 5 & 1.927 & 2.094 & 2.033 & 1,992 & 1.964 & 1.940 & 2.095 & 2.034 & 1.993 & 1.965 & 1,941 \\
\hline & & 1 & 1,086 & 1,055 & 1,033 & 1,019 & 1,006 & 1,087 & 1,055 & 1,034 & 1,019 & 1,007 \\
\hline & 10 & 1,619 & 1,706 & 1,674 & 1,652 & 1,637 & 1,624 & 1,706 & 1,674 & 1,652 & 1,637 & 1,624 \\
\hline & & 1 & 1,054 & 1,034 & 1,020 & 1,011 & 1,003 & 1,054 & 1,034 & 1,021 & 1,011 & 1,003 \\
\hline ร & 15 & 1,496 & 1,558 & 1,535 & 1,519 & 1,508 & 1,498 & 1,558 & 1,535 & 1,519 & 1,508 & 1,499 \\
\hline 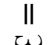 & & 1 & 1,042 & 1,026 & 1,016 & 1,008 & 1,002 & 1,042 & 1,026 & 1,016 & 1,008 & 1,002 \\
\hline . & 20 & 1,425 & 1,475 & 1,457 & 1,444 & 1,435 & 1,427 & 1,475 & 1,457 & 1,444 & 1,435 & 1,427 \\
\hline 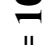 & & 1 & 1,035 & 1,022 & 1,013 & 1,007 & 1,001 & 1,035 & 1,022 & 1,013 & 1,007 & 1,001 \\
\hline & 25 & 1,378 & 1,421 & 1,405 & 1,394 & 1,386 & 1,379 & 1,420 & 1,405 & 1,394 & 1,386 & 1,379 \\
\hline & & 1 & 1,031 & 1,019 & 1,011 & 1,006 & 1,001 & 1,031 & 1,019 & 1,011 & 1,006 & 1,001 \\
\hline & 30 & 1,343 & 1,381 & 1,367 & 1,357 & 1,350 & 1,344 & 1,381 & 1,367 & 1,357 & 1,350 & 1,344 \\
\hline & & 1 & 1,028 & 1,018 & 1,010 & 1,005 & 1,001 & 1,028 & 1,018 & 1,010 & 1,005 & 1,001 \\
\hline
\end{tabular}


Tabela 2 - Medidas de desempenho ajustadas e nominais em função de $m$ e $n$ com $p \in(5 ; 10) \%$; $\varepsilon \in(10 ; 20) \%, \gamma=1,5$ e $\alpha_{\text {nom }}=0,005$.

\begin{tabular}{|c|c|c|c|c|c|c|c|c|c|c|c|c|}
\hline \multicolumn{13}{|c|}{ Medidas de desempenho } \\
\hline \multirow{3}{*}{ قِّة } & \multirow{3}{*}{ n } & $P d_{n o m}$ & \multirow{2}{*}{\multicolumn{5}{|c|}{$\begin{array}{c}\boldsymbol{P d _ { S _ { p } }} \\
\boldsymbol{N M A _ { S _ { p } }}\end{array}$}} & \multirow{2}{*}{\multicolumn{5}{|c|}{$\begin{array}{c}\boldsymbol{P d _ { S _ { c } }} \\
N M A_{S_{c}}\end{array}$}} \\
\hline & & $N M A_{n o n}$ & & & & & & & & & & \\
\hline & & $\forall \mathbf{m}$ & $\mathbf{m}=\mathbf{2 5}$ & 50 & 100 & 200 & 500 & $\mathrm{~m}=\mathbf{2 5}$ & 50 & 100 & 200 & 500 \\
\hline \multirow{12}{*}{$\begin{array}{l}\stackrel{\ominus}{\ominus} \\
\stackrel{0}{\|} \\
\omega \\
\ddot{n} \\
\| \\
\triangleq\end{array}$} & 5 & 0,158 & 0,080 & 0,102 & 0,119 & 0,132 &, 143 & 078 & 0,101 & 0,118 & 131 & 143 \\
\hline & & & & 9,8 & 8,4 & 7,6 & 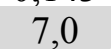 & & 9,9 & 8,5 & & 7,0 \\
\hline & 10 & 0,313 & 0,202 & 0,236 & 0,261 & 0,278 & 0,294 & 0,202 & 0,235 & 0,260 & 0,278 & 0,294 \\
\hline & & 32 & 10 & 4,2 & 3,8 & 36 & 34 & 5,0 & 4,2 & 3,8 & 3, & 3,4 \\
\hline & 15 & 0,456 & 0,3 & 0,372 & 0,400 & 0,419 & & 332 & 0,372 & 0,399 & 0,419 & 0,436 \\
\hline & & 2,2 & & 2, & 2,5 & & & & 2, & 2,5 & & 2,3 \\
\hline & 20 & 0,580 & 0 , &, 498 & 0,526 & 0,545 & & 458 & 0,498 & 0,525 & &, 561 \\
\hline & & & & 2,0 & 1,9 & 18 & & 2,2 & 2,0 & 1,9 & 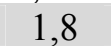 & 1,8 \\
\hline & 25 & 0,683 & 0 & 0,608 & 0,634 & & & 0,570 & 0,608 & 0,634 & & 0,666 \\
\hline & & & & 1,6 & 1,6 & 1 , & & & 1,6 & 1,6 & & 1,5 \\
\hline & 30 & 0,764 & 0 & 0,700 & 0,723 & 0,738 & & & 0,700 & 0,722 & 0,7 & 0,751 \\
\hline & & & & 1,4 & 1,4 & 14 & & 1,5 & 1,4 & 1,4 & 1, & 1,3 \\
\hline \multirow{12}{*}{$\begin{array}{l}\stackrel{\partial}{\hat{\theta}} \\
\| \\
\omega \\
\ddot{\theta} \\
\stackrel{\|}{\|} \\
\Theta\end{array}$} & 5 & & & & 0,128 & & & & 0.114 & 0.128 & & 0.148 \\
\hline & & & & & 7,8 & & & & & 7,8 & & 6,8 \\
\hline & 10 & 0,31 & & ,254 & 0,274 & & & & 0,254 & 0,273 & & 0,300 \\
\hline & & & & & 3,7 & & & & & 3,7 & & 3,3 \\
\hline & 15 & 0,4 & & & 0,414 & & & & & 0,414 & & ,443 \\
\hline & & & & 2 & 2,4 & & & 2,8 & 2,5 & 2,4 & & 2,3 \\
\hline & 20 & 0,580 & & 0,519 & 0,540 & & & 0,488 & & 0,540 & & 0,568 \\
\hline & & & & 1,9 & 1,9 & 1 & & 2,0 & 1,9 & 1,9 & 1 . & 1,8 \\
\hline & 25 & 0,683 & & 0,627 & 0,647 & & & & & 0,647 & & 0,672 \\
\hline & & & & 16 & 1,5 & & & 1,7 & 1,6 & 1,5 & & 1,5 \\
\hline & 30 & 0,764 & 0, & 0,717 & 0,734 & 6 & 6 & ,692 & 0,717 & 0,734 & 0,746 & 0,756 \\
\hline & & & & & 1,4 & & & & & 1,4 & & 1,3 \\
\hline \multirow{12}{*}{ 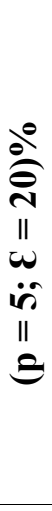 } & 5 & 0,158 & & & 0,124 & & & & & 0,123 & & 0,148 \\
\hline & & & & & 81 & & & & & 8,2 & & 6,8 \\
\hline & 10 & 0 , & & & 0,268 & & & & & 0,268 & & 0,302 \\
\hline & & & & & 3,7 & & & & & 3,7 & & 3,3 \\
\hline & 15 & 0,456 & & 0,381 & 0,409 & & & 0,341 & 0,380 & 0,408 & & 0,445 \\
\hline & & & & & 24 & & & & & 2,4 & & 2,2 \\
\hline & 20 & 0,580 & 0 &, 507 & 0,535 & & & & 0,507 & 0,535 & & 0,571 \\
\hline & & & & & 1,9 & & & & & 1,9 & & 1,8 \\
\hline & 25 & 0,683 & 0 & 0,617 & 0,643 & & & 579 & & 0,642 & & 0,675 \\
\hline & & & & & & & & & & & & \\
\hline & 30 & 0,764 & & 07 & 0,730 & 0, & & 74 & 0 & 0,730 & 0,7 & 0,758 \\
\hline & & & & & 14 & & & & 1 & 1,4 & 1 & 1,3 \\
\hline \multirow{12}{*}{ 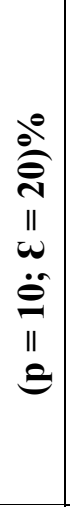 } & 5 & 0.15 & & & 0.133 & & & תم & & 0.133 & & 0153 \\
\hline & & & & 0,4 & 1, & 1, & & & 0, & 7,5 & & 6,5 \\
\hline & 10 & 0,313 & 0,2 & 0,262 & 0,282 & 0,296 & 0,3 & 0,234 & 0,262 & 0,281 & 0,295 & 0,308 \\
\hline & & & & & 8 & & & & & 3,6 & & 3,2 \\
\hline & 15 & 0,456 & 0,370 & 0,402 & 0,423 & 0,439 & & 0,371 & 0,401 & 0,423 & & 0,452 \\
\hline & & & & & 2 , & 2 & & & & 0 & & 2,2 \\
\hline & 20 & 0,580 & 0,49 & 0,528 & 0,549 & 0,5 & & 0,497 & 0,528 & 0,549 & & 0,577 \\
\hline & & & & & & & & & & & & 1,7 \\
\hline & 25 & 0,683 & 0,60 & 0636 & 0,656 & 0,6 & & 0,608 & 0,636 & 0,655 & 0,669 & 0,680 \\
\hline & & & & 1 & 1 & & & 16 & 16 & 1,5 & 15 & 15 \\
\hline & 30 & 0,7 & 0 & 0,725 & 0,742 & 0,7 & & 0,700 & 0,725 & 0,742 & 0,753 & 0,763 \\
\hline & & 1,3 & 1,4 & 1,4 & 1,3 & 1,3 & 1,3 & 1,4 & 1,4 & 1,3 & 1,3 & 1,3 \\
\hline
\end{tabular}


Figura 1 - Limites de controle, poder e NMA ajustados.

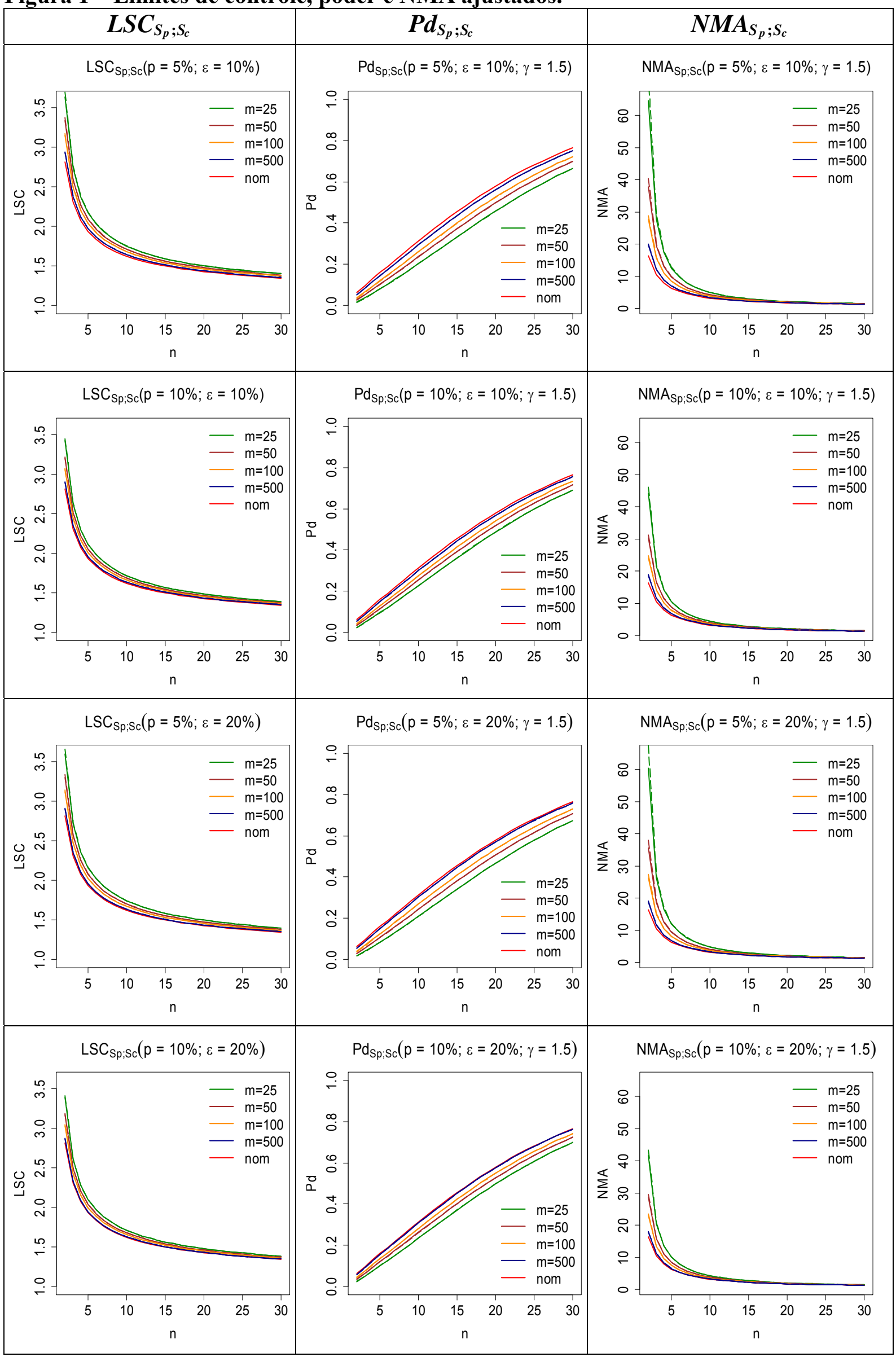


Tabela 3 - Variação relativa entre as medidas de desempenho ajustadas e as nominais em função de $m$ e $n$ com $p \in(5 ; 10) \% ; \varepsilon \in(10 ; 20) \%, \gamma=1,5$ e $\alpha_{\text {nom }}=0,005$.

\begin{tabular}{|c|c|c|c|c|c|c|c|c|c|c|c|c|}
\hline \multicolumn{13}{|c|}{ Variação relativa } \\
\hline \multirow{3}{*}{$\begin{array}{l}\underline{\vdots} \\
\ddot{\Xi}\end{array}$} & \multirow{3}{*}{$\mathrm{n}$} & $P d_{n o m}$ & \multirow{2}{*}{\multicolumn{5}{|c|}{ 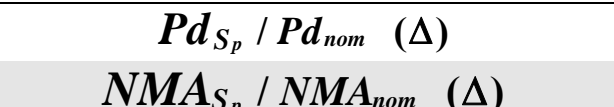 }} & \multicolumn{5}{|c|}{$P d_{S_{c}} / P d_{n o m}(\Delta)$} \\
\hline & & $\Delta$ & & & & & & \multicolumn{5}{|c|}{$N M A_{S_{c}} / N M A_{n o m}(\Delta)$} \\
\hline & & $\forall \mathbf{m}$ & $m=25$ & $\mathbf{5 0}$ & 100 & 200 & 500 & $\mathrm{~m}=\mathbf{2 5}$ & $\mathbf{5 0}$ & 100 & 200 & 500 \\
\hline \multirow{12}{*}{$\begin{array}{l}\stackrel{\partial}{\varrho} \\
\stackrel{\varrho}{\|} \\
\omega \\
\ddot{n} \\
\| \\
\varrho\end{array}$} & \multirow[t]{2}{*}{5} & 1 & 0,502 & 0,643 & 0,751 & 0,832 & 0,906 & \begin{tabular}{|l|l}
0,494 \\
\end{tabular} & 0,635 & 0.745 & 0,827 & 0,903 \\
\hline & & 1 & 2,0 & 1,6 & 1,3 & 1,2 & 1,1 & 2,0 & 1,6 & 1,3 & 1,2 & 1,1 \\
\hline & \multirow[t]{2}{*}{10} & 1 & 0,648 & 0,755 & 0,834 & 0,890 & 0,940 & 0,645 & 0,753 & 0,832 & 0,888 & 0,939 \\
\hline & & 1 & 1,5 & 1,3 & 1,2 & 1,1 & 1,1 & 1,6 & 1,3 & 1,2 & 1,1 & 1,1 \\
\hline & \multirow[t]{2}{*}{15} & 1 & 0,730 & 0,816 & 0,877 & 0,920 & 0,958 & 0,729 & 0,815 & 0,876 & 0,919 & 0,957 \\
\hline & & 1 & 1,4 & 1,2 & 1,1 & 1,1 & 1,0 & 1,4 & 1,2 & 1,1 & 1,1 & 1,0 \\
\hline & \multirow[t]{2}{*}{20} & 1 & 0,790 & 0,859 & 0,907 & 0,940 & 0,968 & 0,789 & 0,858 & 0,906 & 0,939 & 0,968 \\
\hline & & 1 & 1,3 & 1,2 & 1,1 & 1,1 & 1,0 & 1,3 & 1,2 & 1,1 & 1,1 & 1,0 \\
\hline & 25 & 1 & 0,835 & 0,891 & 0,928 & 0,954 & 0,976 & 0,835 & 0,890 & 0,928 & 0,954 & 0,976 \\
\hline & & 1 & 1,2 & 1,1 & 1,1 & 1,0 & 1,0 & 1,2 & 1,1 & 1,1 & 1,0 & 1,0 \\
\hline & \multirow[t]{2}{*}{30} & 1 & 0,871 & 0,916 & 0,945 & 0,965 & 0,982 & 0,871 & 0,915 & 0,945 & 0,965 & 0,982 \\
\hline & & 1 & 1,1 & 1,1 & 1,1 & 1,0 & 1,0 & 1,1 & 1,1 & 1,1 & 1,0 & 1,0 \\
\hline \multirow{12}{*}{ 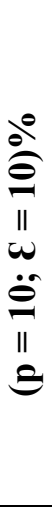 } & \multirow[t]{2}{*}{5} & 1 & 0.601 & 0.721 & 0.810 & 0.875 & 0.934 & 0.599 & 0.718 & 0.807 & 0.872 & 0.932 \\
\hline & & 1 & 1,7 & 1,4 & 1,2 & 1,1 & 1,1 & 1,7 & 1,4 & 1,2 & 1,1 & 1,1 \\
\hline & 10 & 1 & 0,725 & 0,813 & 0,875 & 0,920 & 0,960 & 0,726 & 0,812 & 0,874 & 0,919 & 0,959 \\
\hline & & 1 & 1,4 & 1,2 & 1,1 & 1,1 & 1,0 & 1,4 & 1,2 & 1,1 & 1,1 & 1,0 \\
\hline & 15 & 1 & 0,793 & 0,861 & 0,909 & 0,942 & 0,972 & 0,794 & 0,861 & 0,909 & 0,942 & 0,971 \\
\hline & & 1 & 1,3 & 1,2 & 1,1 & 1,1 & 1,0 & 1,3 & 1,2 & 1,1 & 1,1 & 1,0 \\
\hline & 20 & 1 & 0,841 & 0,894 & 0,931 & 0,957 & 0,979 & 0,841 & 0,894 & 0,931 & 0,957 & 0,979 \\
\hline & & 1 & 1,2 & 1,1 & 1,1 & 1,0 & 1,0 & 1,2 & 1,1 & 1,1 & 1,0 & 1,0 \\
\hline & 25 & 1 & 0,876 & 0,919 & 0,948 & 0,967 & 0,985 & 0,877 & 0,919 & 0,948 & 0,967 & 0,984 \\
\hline & & 1 & 1,1 & 1,1 & 1,1 & 1,0 & 1,0 & 1,1 & 1,1 & 1,1 & 1,0 & 1,0 \\
\hline & 30 & 1 & 0,904 & 0,938 & 0,960 & 0,975 & 0,988 & 0,905 & 0,938 & 0,960 & 0,975 & 0,988 \\
\hline & & 1 & 1,1 & 1,1 & 1,0 & 1,0 & 1,0 & 1,1 & 1,1 & 1,0 & 1,0 & 1,0 \\
\hline & 5 & 1 & 0,526 & 0,670 & 0,781 & 0,863 & 0,939 & 0,517 & 0,663 & 0,775 & 0,859 & 0,936 \\
\hline & & 1 & 1,9 & 1,5 & 1,3 & 1,2 & 1,1 & 1,9 & 1,5 & 1,3 & 1,2 & 1,1 \\
\hline & 10 & 1 & 0,669 & 0,779 & 0,858 & 0,915 & 0,966 & 0,667 & 0,776 & 0,856 & 0,914 & 0,965 \\
\hline$\stackrel{\circ}{\partial}$ & & 1 & 1,5 & 1,3 & 1,2 & 1,1 & 1,0 & 1,5 & 1,3 & 1,2 & 1,1 & 1,0 \\
\hline ฮి & 15 & 1 & 0,750 & 0,836 & 0,897 & 0,940 & 0,978 & 0,748 & 0,835 & 0,896 & 0,939 & 0,977 \\
\hline$\|$ & & 1 & 1,3 & 1,2 & 1,1 & 1,1 & 1,0 & 1,3 & 1,2 & 1,1 & 1,1 & 1,0 \\
\hline$\underline{\omega}$ & 20 & 1 & 0,806 & 0,875 & 0,923 & 0,956 & 0,984 & 0,805 & 0,874 & 0,922 & 0,955 & 0,984 \\
\hline$n$ & & 1 & 1,2 & 1,1 & 1,1 & 1,0 & 1,0 & 1,2 & 1,1 & 1,1 & 1,0 & 1,0 \\
\hline 3 & 25 & 1 & 0,849 & 0,904 & 0,941 & 0,967 & 0,989 & 0,849 & 0,904 & 0,941 & 0,967 & 0,988 \\
\hline & & 1 & 1,2 & 1,1 & 1,1 & 1,0 & 1,0 & 1,2 & 1,1 & 1,1 & 1,0 & 1,0 \\
\hline & 30 & 1 & 0,882 & 0,926 & 0,955 & 0,975 & 0,992 & 0,882 & 0,926 & 0,955 & 0,975 & 0,992 \\
\hline & & 1 & 1,1 & 1,1 & 1,0 & 1,0 & 1,0 & 1,1 & 1,1 & 1,0 & 1,0 & 1,0 \\
\hline & 5 & 1 & 0,628 & 0,750 & 0,841 & 0,908 & 0,968 & 0,625 & 0,747 & 0,838 & 0,905 & 0,965 \\
\hline & & 1 & 1,6 & 1,3 & 1,2 & 1,1 & 1,0 & 1,6 & 1,3 & 1,2 & 1,1 & 1,0 \\
\hline & 10 & 1 & 0,749 & 0,837 & 0,900 & 0,945 & 0,985 & 0,749 & 0,836 & 0,899 & 0,944 & 0,985 \\
\hline$\stackrel{\circ}{\check{\sigma}}$ & & 1 & 1,3 & 1,2 & 1,1 & 1,1 & 1,0 & 1,3 & 1,2 & 1,1 & 1,1 & 1,0 \\
\hline తి & 15 & 1 & 0,813 & 0,881 & 0,929 & 0,962 & 0,992 & 0,814 & 0,881 & 0,929 & 0,962 & 0,991 \\
\hline$\underset{\omega}{\|}$ & & 1 & 1,2 & 1,1 & 1,1 & 1,0 & 1,0 & 1,2 & 1,1 & 1,1 & 1,0 & 1,0 \\
\hline$\ddot{\theta}$ & 20 & 1 & 0,857 & 0,910 & 0,947 & 0,973 & 0,995 & 0,858 & 0,911 & 0,947 & 0,973 & 0,995 \\
\hline$=$ & & 1 & 1,2 & 1,1 & 1,1 & 1,0 & 1,0 & 1,2 & 1,1 & 1,1 & 1,0 & 1,0 \\
\hline$=$ & 25 & 1 & 0,890 & 0,932 & 0,960 & 0,980 & 0,997 & 0,890 & 0,932 & 0,960 & 0,980 & 0,997 \\
\hline & & 1 & 1,1 & 1,1 & 1,0 & 1,0 & 1,0 & 1,1 & 1,1 & 1,0 & 1,0 & 1,0 \\
\hline & 30 & 1 & 0,915 & 0,948 & 0,970 & 0,985 & 0,998 & 0,916 & 0,948 & 0,970 & 0,985 & 0,998 \\
\hline & & 1 & 1,1 & 1,1 & 1,0 & 1,0 & 1,0 & 1,1 & 1,1 & 1,0 & 1,0 & 1,0 \\
\hline
\end{tabular}


Figura 2 - Variação relativa dos limites de controle, poder e NMA ajustados.

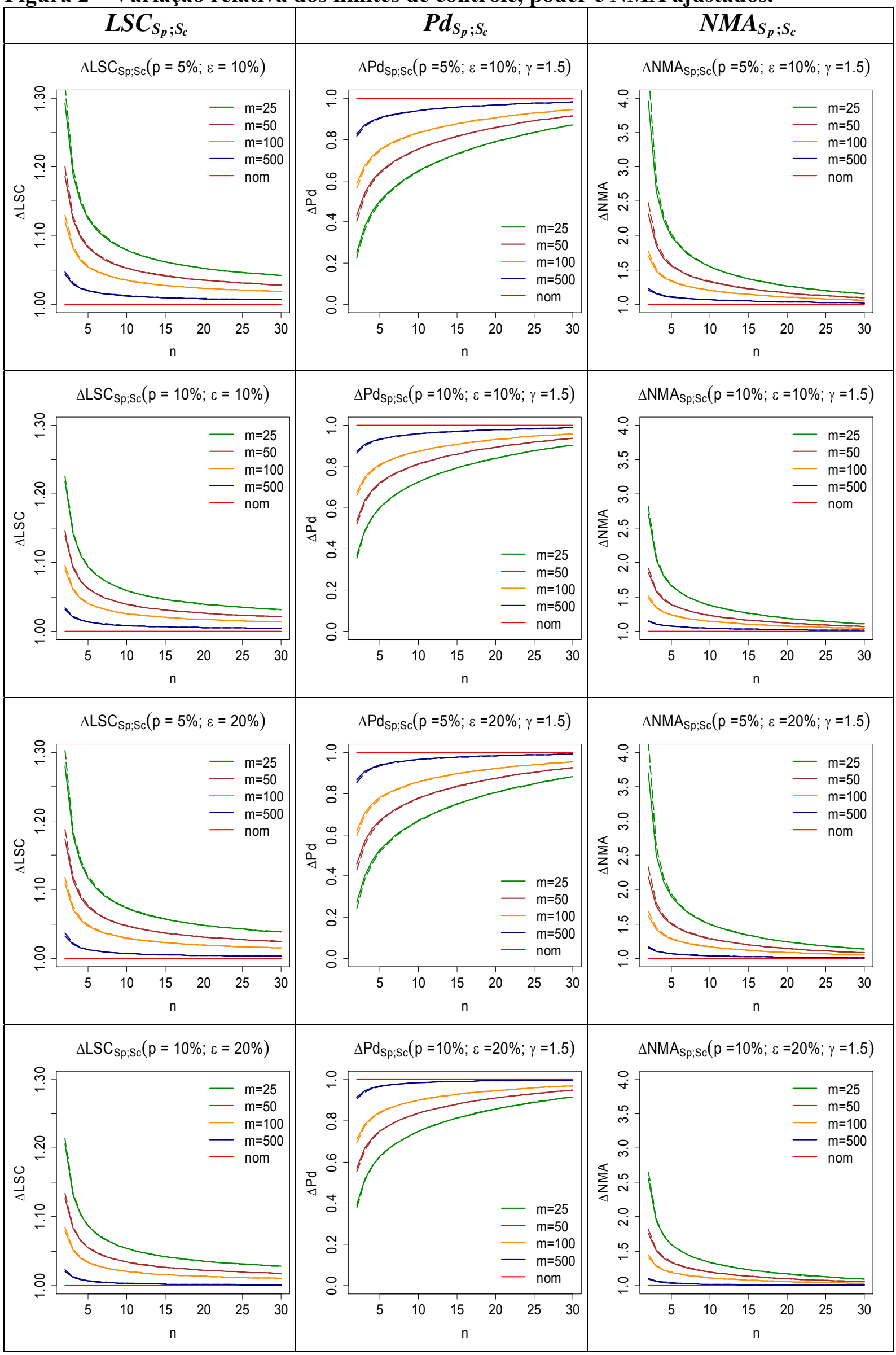


Quanto maior o deslocamento sofrido pelo desvio-padrão do processo maior o desempenho do gráfico na detecção de um processo fora de controle e também mais próximas estarão as medidas ajustadas das medidas nominais. Para exemplificar apresentamos a seguir as curvas características de operação (CCO’s) - Figura 3 - que correspondem aos poderes ajustados em função de $\gamma$ para $m$ e $n$ selecionados com $(p=5 \% ; \varepsilon=10 \%)$. Podemos ver que mesmo com $(m=25 ; n=5)$ o $P d_{S_{p}}$ corresponde a $72 \%$ do $P d_{n o m}$ se $\gamma=2$, aumentando para $81 \%$ e $87 \%$ quando $m=50$ e $m=100$, respectivamente. Na tabela 4 apresentamos a variação relativa entre o $P d_{S_{p}}$ e o $P d_{\text {nom }}$ (os valores com relação ao $P d_{S_{c}}$ são próximos).

Figura 3 - Curvas características de operação para $m$ e $n$ selecionados $\operatorname{com}(p ; \varepsilon)=(5 ; 10) \%$.

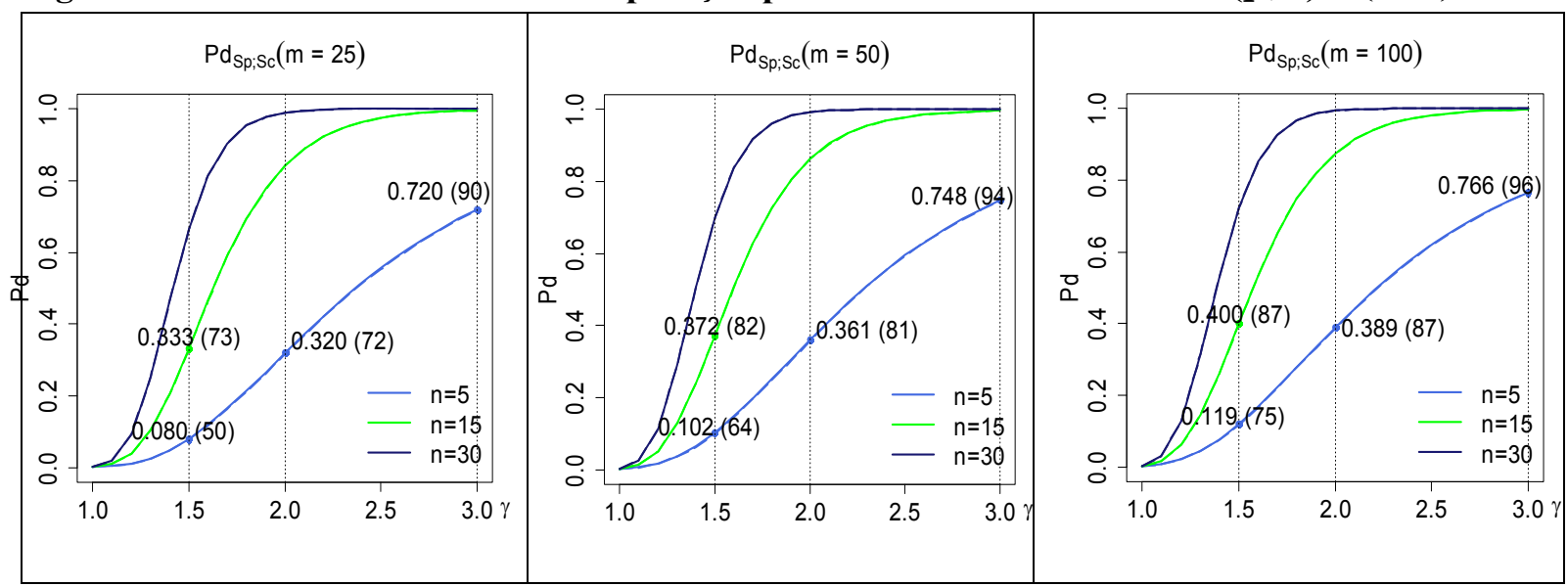

Tabela 4 - Variação relativa entre o $P d_{s_{p}}$ e o $P d_{n o m}$ em função de $\gamma \operatorname{com}(p ; \varepsilon)=(5 ; 10) \%$.

\begin{tabular}{c|ccc|ccc|ccc}
\hline \multirow{2}{*}{$\mathbf{n}$} & \multicolumn{3}{|c|}{$\boldsymbol{P d}_{\boldsymbol{S}_{\boldsymbol{p}}}(\boldsymbol{m}=\mathbf{2 5})$} & \multicolumn{3}{c|}{$\boldsymbol{P d}_{\boldsymbol{S}_{\boldsymbol{p}}}(\boldsymbol{m}=\mathbf{5 0})$} & \multicolumn{3}{c}{$\boldsymbol{P d}_{\boldsymbol{S}_{\boldsymbol{p}}}(\boldsymbol{m}=\mathbf{1 0 0})$} \\
\cline { 2 - 9 } & $\gamma=\mathbf{1 , 5}$ & $\gamma=\mathbf{2}$ & $\gamma=\mathbf{3}$ & $\gamma=\mathbf{1 , 5}$ & $\gamma=\mathbf{2}$ & $\gamma=\mathbf{3}$ & $\gamma=\mathbf{1 , 5}$ & $\gamma=\mathbf{2}$ & $\gamma=\mathbf{3}$ \\
\hline $\mathbf{5}$ & 0,51 & 0,72 & 0,90 & 0,64 & 0,81 & 0,91 & 0,75 & 0,87 & 0,96 \\
$\mathbf{1 5}$ & 0,73 & 0,94 & 1 & 0,82 & 0,96 & 1 & 0,88 & 0,97 & 1 \\
$\mathbf{3 0}$ & 0,87 & 1 & 1 & 0,93 & 1 & 1 & 0,96 & 1 & 1 \\
\hline
\end{tabular}

\section{CONCLUSÃO}

Neste artigo apresentamos os limites de controle ajustados para o gráfico de $S$ com parâmetro estimado por $S_{p}$ e $S_{c}$ e com baixa probabilidade de o risco $\alpha_{\text {real }}$ ser ultrapassado pelo risco $\alpha_{\text {tol }}$. De acordo com os resultados, observamos que, com $m$ e $n$ muito baixos, por exemplo, em torno de $m=25$ e $n=5$, teremos limites de controle ajustados que serão, no mínimo, $12 \%$ maiores que os limites nominais. Estes têm sido o número e tamanho de amostras preliminares sugeridos pela maior parte dos manuais de CEP a fim de que os parâmetros sejam estimados e os limites de controle estabelecidos. Por outro lado, a partir de uma combinação entre as variáveis $m, n, p$ e $\varepsilon$, o usuário pode obter limites de controle ajustados muito próximo aos nominais, de modo que o desempenho do gráfico de $S$ na sinalização de aumentos no desvio-padrão do processo se encontre muito próximo ao "desempenho nominal", mesmo no casos de pequenos aumentos. Por exemplo, se $m=25$ e $n=15$, os limites ajustados são $6 \%$ maiores que o limite de controle nominal, com poderes ajustados correspondendo a $73 \%$ do poder nominal, se considerarmos $p=5 \%$ e $\varepsilon=10 \%$ com $\gamma=1.5$. A escolha dos valores de $m, p$ e $\varepsilon$ envolve as preferências subjetivas do usuário, mas as tabelas aqui apresentadas permitem-lhe avaliar as alternativas de acordo com essas suas preferências. De qualquer modo, este trabalho apenas abre o caminho para o necessário prosseguimento das pesquisas nessa questão tão relevante para o praticante de CEP. 


\section{REFERÊNCIAS BIBLIOGRÁFICAS}

[1] ALBERS, W.; KALLENBERG, W.C.M. Are Estimated Control Charts in Control? Statistics 38(1), pp. 67-79. 2004.

[2] CHAKRABORTI, S. Run Length, Average Run Length and False Alarm Rate of Shewhart $\overline{\boldsymbol{X}}$ Chart: Exact Derivations by Conditioning. Journal of Applied Statistics, 33(4), pp. 439-459. 2000.

[3] CHAKRABORTI, S. Parameter Estimation and Design Considerations in Prospective Applications of the $\overline{\boldsymbol{X}}$ Chart. Communications in Statistics - Simulation and Computation 29(1), pp. 61-81. 2006.

[4] CHAKRABORTI, S. Run Length Distribution and Percentiles: The Shewhart $\overline{\boldsymbol{X}}$ Chart with Unknown Parameters. Quality Engineering 19, pp. 119-127. 2007.

[5] COSTA, F. B. C.; EPPRECHT, E. K.; CARPINETTI, L. C. R. Controle Estatístico de Qualidade. 2. ed. São Paulo: Atlas, 334p. 2012.

[6] EPPRECHT, E. K.; LOUREIRO, L. D. Effect of the Number and Size of the Initial Samples on the Performance of the S Chart. Joint Statistical Meeting - JSM 2012, 2012, San Diego, CA. JSM Proceedings. Alexandria, VA, EUA: American Statistical Association, p. 1571-1584. 2012.

[7] EPPRECHT, E. K.; LOUREIRO, L.D.; CHAKRABORTI, S. Effect of the Number and Size of the Phase I Sample on the Phase II Performance of $\mathbf{S}^{2}$ and $\mathbf{S}$ Control Charts. Journal of Quality Technology, 47(2), 2015.

[8] LOUREIRO, L.D. Efeito do Número e Tamanho das Amostras Iniciais sobre o Desempenho do Gráfico de $\mathrm{S}$ e sobre a Estimativa da Capacidade do Processo. Dissertação de mestrado, Departamento de Engenharia Industrial, Pontifícia Universidade Católica do Rio de Janeiro. 2008.

[9] MAHMOUD, M. A.; HENDERSON, G. R.; EPPRECHT, E. K.; WOODALL, W. H. Estimating the Standard Deviation in Quality Control Applications. Journal of Quality Technology, 42(4), pp. 348-357. 2010.

[10] MONTGOMERY, D.C. Introduction to Statistical Quality Control, 7ed. John Wiley \& Sons, Hoboken, NJ. 513p. 2011.

[11] WOODALL, W. H; MONTGOMERY, D. C. Research Issues and Ideas in Statistical Process Control. Journal of Quality Technology, 31(4), 376-386. 1999. 